\title{
Atherosclerosis and venous thromboembolism similarities
}

\author{
Anetta Undas \\ Institute of Cardiology, Jagiellonian University Medical College, John Paul II Hospital, Krakow, Poland
}

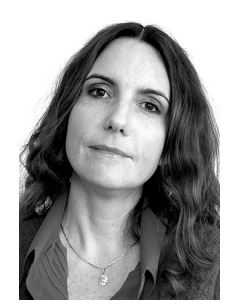

INTRODUCTION

Venous thromboembolism (VTE), encompassing deep vein thrombosis (DVT) and pulmonary embolism (PE), and atherothrombosis have long been considered to be separate entities with distinct pathogenic mechanisms.

The traditional rationale for this concept has been based on: (1) pathologic data showing platelet-rich thrombi in the arteries in contrast to 'red clots' observed in veins; (2) clinical data on a substantial efficacy of antiplatelet agents in the prevention of arterial thromboembolic events in contrast to negligible benefits from these drugs in patients with VTE; (3) experimental data demonstrating diffuse inflammatory infiltrates, extracellular cholesterol deposits, neovessel formation and calcification within advanced atherosclerotic lesions within the arterial wall in contrast to minor lesions largely within the endothelial layer of the thrombosed veins.

A key component of this concept is the assumption that deranged local processes occurring inside the vessel wall account for arterial thrombosis with a minor contribution by blood-borne abnormalities, whereas a combination of extrinsic factors ranging from venous blood stasis to hypercoagulable blood that act on a normal wall of a vein determine the occurrence of venous thrombosis.

Experimental data derived from biochemical laboratories, cell cultures and animal models has shown however many similarities in the pathophysiology of arterial and venous thrombosis [1]. Coagulation pathways and their regulation are in fact identical in arteries and veins. Blood circulating in the two types of vessels is the same in terms of coagulation proteins. All kinds of thrombosis are triggered with the same major physiological initiator of blood coagulation, i.e. tissue factor (TF) [2]. Finally, thrombus composition assessed following thrombectomy in patients with ST-segment elevation acute myocardial infarction (MI) is frequently hard to differentiate from that of thrombi obtained from thrombosed veins, as evidenced by the fact that fibrin represents the largest por- tion of the thrombotic mass in both types of vessels $[3,4]$. Despite the key role of activated platelets as a driving factor of thrombus formation under high shear stress conditions, there is convincing evidence for a significant contribution of thrombin and blood coagulation proteins in arterial thrombosis, as well as for their expression in macrophages and other cells within atherosclerotic lesions [5].

Until the beginning of the current century, there was no clinical data to support basic science findings indicating that thrombus formation in most vessels follows the same pattern and once deranged haemostasis tilted toward thrombosis results in increased risk of thromboembolic events putting in danger both the arterial and venous beds. Within the last decade, accumulating evidence has indicated that patients following VTE are at risk of MI or ischaemic stroke and vice versa. However a lot of experimental and clinical questions are to be answered including those as to whether all affected patients are indeed prone to develop the other type of thrombosis and which diagnostic and therapeutic strategy should be adopted in such a clinical setting.

This review summarises the most important clinical and experimental data on the association between arterial and venous thrombosis.

\section{EPIDEMIOLOGICAL DATA}

Does VTE predispose to atherothrombosis?

Prandoni et al. [6] were the first to report that VTE may predispose to atherosclerosis. In a cross-sectional case-control study published in 2003, they showed using carotid ultrasound scanning that the prevalence of carotid plaques was higher in patients with idiopathic VTE but with no history of symptomatic atherosclerosis (47\%) than in those with provoked VTE $(27 \%)$ or in age- and sex-matched controls free of thrombosis (32\%). These differences remained significant after adjusting for risk factors for atherosclerosis and thrombophilia.

Growing evidence indicates that VTE increases the risk of subsequent arterial thrombotic events, primarily $\mathrm{MI}$ or ischaemic stroke [7-10]. In the largest of these studies published to 
date, Sorensen et al. [10] performed a retrospective analysis of nationwide Danish medical databases involving more than 40,000 patients with VTE and 160,000 controls followed for 20 years. Comparison of the risks of $\mathrm{MI}$ and stroke showed that after excluding the patients with arterial hypertension, coronary heart disease (CHD), stroke or transient ischaemic attack (TIA), subjects with DVT during the first year after the VTE event had a relative risk (RR) for $\mathrm{MI}$ of 1.60 (95\% confidence interval [Cl] 1.35-1.91) and for stroke of 2.19 (95\% Cl 1.85-2.60), while for patients with PE the corresponding RR values were for $\mathrm{Ml} 2.60$ (95\% Cl 2.14-3.14) and for stroke 2.93 (95\% Cl 2.34-3.66). Increased risks of MI and stroke were also observed during the subsequent 20 years of follow-up. Interestingly, these relative risks were similar for those with idiopathic VTE and those with VTE associated with malignancy, trauma, surgery or pregnancy [10]. The increased risk of MI and stroke was highest in the first year after diagnosis of VTE despite the standard anticoagulant treatment of 3-6 months' duration [10].

The risk of death from cardiovascular causes was four-fold higher in patients with residual thrombus in the veins affected by DVT than in those with full recanalisation [11].

In a meta-analysis of six studies on arterial cardiovascular events following a VTE episode published in 2010, Becattini et al. [12] reported that the risk of arterial cardiovascular events was approximately $90 \%$ higher in patients with both unprovoked (incidence rate ratio [IRR] 1.87; 95\% Cl 1.32-2.65) and provoked VTE (IRR 1.86, 95\% Cl 1.19-2.89) vs. the controls.

On the other hand, VTE may directly cause arterial thrombosis, as evidenced by the well-known phenomenon of 'paradoxical' embolism through a right-to-left intracardiac shunt leading to ischaemic cerebrovascular events [13].

The latest evidence on a possible association between VTE and arterial thrombosis came from the prospective cohort Tromso study (screening visits in 1994-1995) and the Diet, Cancer and Health Study (screening visits 1993-1997) involving approximately 82,000 subjects aged 24-97 years without a previous history of VTE or arterial thrombotic diseases at inclusion. In 2013, Lind et al. [14] demonstrated that during a median follow-up of 12.2 years, subjects suffering from a VTE event had a $35 \%$ higher risk of a future arterial event (adjusted hazard ratio [HR] 1.35; 95\% Cl 1.09-1.66). Interestingly, women (but not men) and subjects below 65 years of age (but not older) with incident VTE had a 3-4-fold increased risk of future $\mathrm{MI}$ and ischaemic stroke, with as little as $1 \%$ of the arterial thrombotic events attributed to VTE [14].

To sum up, several lines of evidence support the view that patients who have developed DVT or PE, particularly when unprovoked, have a heightened risk of various clinical manifestations of atherothrombosis with the largest prevalence of MI. However, this concept is exclusively based on findings in selective patient cohorts or population-based prospective registries.

\section{Does atherosclerosis precede VTE?}

Several clinical studies have reported that patients with symptomatic atherothrombotic disease, more precisely those who experienced $\mathrm{MI}$ or ischaemic stroke associated with carotid stenosis, are at an increased risk of incident VTE [15].

Autopsy studies based on objectively documented thrombotic occlusions indicate that also uncommon locations of arterial thrombosis other than coronary or carotid artery thrombosis can be encountered in patients with a prior history of VTE. Eliasson et al. [16] reported a higher prevalence of VTE in patients with atherothrombotic episodes involving the carotid, cranial, visceral, iliac and femoral arteries and the aorta, but not of coronary arteries.

Of note, there is no evidence that subclinical atherosclerosis, largely evidenced by increased intima-media thickness measured in carotid arteries or by the presence of asymptomatic atherosclerotic plaques on ultrasound scanning, is associated with an increased risk of VTE. The two population-based cohort studies performed in subjects aged 45-64 years and in the elderly failed to demonstrate any associations of subclinical atherosclerosis with the onset of VTE after a mean follow-up of 12.5 years or a lower VTE risk during a median follow-up of 11.7 years, respectively $[17,18]$.

Atherosclerosis and arterial thromboembolism may precede VTE, as reported in a few studies [19]. Symptomatic atherosclerosis is observed in about $20 \%$ of VTE patients who are typically older and are more likely to have immobility, prior heart failure, chronic lung disease, cerebrovascular disease, and chronic kidney disease compared to the remaining patients; patients with VTE and coexistent atherosclerosis receive less frequently thromboprophylaxis when hospitalised for causes unrelated to VTE [19].

The best example of such an association is an increased risk of VTE, particularly DVT, following MI or stroke, which probably results from venous stasis in immobilised patients and a transient systemic inflammation and hypercoagulability combined with platelet activation following tissue injury.

To sum up, symptomatic cardiovascular events, particularly MI, can significantly increase the probability of developing incident VTE, whereas subclinical atherosclerosis is not predictive of VTE during a long-term follow-up.

\section{Are risk factors for atherothrombosis and VTE identical?}

The currently prevalent view assumes that both clinical conditions share a substantial proportion of known risk factors, both classic and emerging cardiovascular as well as thrombotic risk factors, both genetically determined and transient environmental factors. However, the available evidence indicates that some risk factors, predominantly a subset of VTE risk factors, are peculiar to either condition. 


\section{Cardiovascular risk factors and the risk of VTE}

In 2008, Ageno et al. [20] published the largest meta-analysis of 21 case-control and cohort studies totalling more than 63,000 patients that demonstrated the association of cardiovascular risk factors with VTE. Obesity (odds ratio [OR] 2.33; 95\% Cl 1.68-3.24), arterial hypertension (OR 1.51; 95\% $\mathrm{Cl} 1.23-1.85)$ and diabetes mellitus (OR 1.42; 95\% Cl 1.12-1.77) were significantly associated with an increased risk of VTE. Surprisingly, no such associations were found for current smoking (OR 1.15; 95\% Cl 0.92-1.44). The same holds true for hypercholesterolaemia (OR 1.16, 95\% Cl, 0.67-2.02) [20].

Some controversy exists around the impact of dyslipidaemia on VTE. Compelling evidence indicates that low HDL cholesterol is associated with increased risk of VTE [21], which has been confirmed in the analysis by Ageno et al. [20]. Hypercholesterolaemia together with arterial hypertension, impaired fasting glucose, and abdominal obesity, can independently predict idiopathic early-onset VTE [22]. Large cohort studies have failed to show any association between lipid profile and the risk of VTE during follow-up [20].

Novel or emerging cardiovascular risk factors can also predict VTE. For example, it has been shown that patients with microalbuminuria are at increased risk of first and recurrent VTE [23]. Furthermore, increased C-reactive protein (CRP) in circulating blood has been shown to be associated with VTE in the general population [24].

To sum up, age, obesity and diabetes have been consistently demonstrated to be associated with higher risk of VTE. Less convincing data links VTE with current smoking and dyslipidaemia. It should be also highlighted that the significant associations between the two types of thromboembolism or their risk factors or markers do not necessarily mean a cause-effect relationship.

\section{Thrombotic risk factors and atherothrombosis} Genetic VTE risk factors, including factor V Leiden (FVL), prothrombin G20210A variant, deficiencies in natural anticoagulants, i.e. antithrombin, protein C (PC), protein S (PS), positive family history, as well as transient or environmental risk factors, including surgery, trauma, prolonged immobility, pregnancy, hormone replacement therapy and oral contraceptive use, obesity, malignancy, are all consistently associated with VTE [25].

Most investigators do not recommend thrombophilia screening in patients with arterial thrombosis [26]. The strongest evidence suggesting that inherited thrombophilia could predispose to arterial thrombosis is based on a meta-analysis of 191 studies involving a total of more than 66,000 coronary artery disease cases and 90,000 controls [27]. In this study published in 2006, Ye et al. [27] reported increased risk of $\mathrm{MI}$ in carriers of FVL and prothrombin G20210A variant
(RR 1.17; 95\% Cl 1.08-1.28 and RR 1.31; 95\% Cl 1.12-1.52, respectively).

Uncommon thrombophilic factors, for example antithrombin deficiency, may heighten the risk of arterial thrombosis, particularly in young individuals as shown also in Polish patients [28, 29]. A large family cohort study demonstrated that hereditary PC (PC antigen $<63 \%$ and/or activity < 64\%) or PS (PS antigen $<68 \%$ ) deficiency is associated with increased risk of arterial thrombosis, defined as $\mathrm{MI}$, ischaemic stroke or TIA, before the age of 55 years, but not in older subjects [30]. In most such cases, arterial thromboembolic events occur in subjects with low to moderate atherosclerotic plaque burden, indicating a significant impact of a hypercoagulable state usually enhanced by a transient factor, for example infection. It should be highlighted that in some VTE patients with thrombophilia, long-term anticoagulation is effective in preventing arterial thromboembolism, thus reducing the prevalence of ischaemic stroke if anticoagulation is started early after a VTE episode, which hampers the interpretation of observational data on the prevalence of $\mathrm{MI}$ or stroke in thrombophilic subjects. Nevertheless, several experts recommend the determination of thrombophilic factors in stroke and/or Ml patients below 45-50 years of age, especially in the absence of the established cardiovascular risk factors.

There is no doubt that antiphospholipid syndrome (APS), a common systemic autoimmune disease characterised by the presence of detectable antiphospholipid antibodies, is associated with both VTE, which is reported in about $70 \%$ of APS patients, and arterial thrombosis, mainly cerebrovascular ischaemic events, which occurs in $20 \%$ of APS patients [31]. Of note, other arterial events are also reported in this thrombophilia and the incidence of $\mathrm{MI}$ is higher among APS patients aged 45 or below than in the general population [32].

Links between acquired transient VTE risk factors and arterial thrombosis are less consistent. For example, a meta-analysis of 31 randomised controlled trials from 2008 showed that hormonal replacement therapy is associated with an increase in VTE (OR 2.05; 95\% Cl 1.44-2.92) and stroke (OR 1.32; 95\% Cl 1.14-1.53) but not in CHD [33].

To sum up, currently inherited thrombophilia has a slight or no predictive value of atherothrombosis in the general population. Acquired prothrombotic factors, in particular APS, increase the risk of $\mathrm{MI}$ and stroke.

\section{PATHOPHYSIOLOGICAL BACKGROUND}

Mechanisms underlying an association between arterial and venous thromboembolism are not fully elucidated. It is known that endothelial cell activation and/or damage, combined with platelet activation are involved in the initiation of thrombus formation in veins and arteries, though their relative contribution differs with regard to the vessel type. A Virchow's triad can also be perceived as applicable in all types of thrombosis, with the largest contribution of disturbances in venous blood 
flow and hypercoagulability for VTE and that of endothelial damage (or rather that of the intima) for MI. However, current evidence challenges this traditional view. Complex fluid mechanics in the valve pockets and blood stasis can lead to endothelial dysfunction and hypoxia which both may contribute to thrombogenesis [34]. Robust experimental data has shown that platelet activation and neutrophil recruitment with the formation of neutrophil extracellular traps are also involved in the pathogenesis of DVT [34-36]. A role of these blood cell-related mechanisms reinforces for example the observation that in transgenic mice expressing low levels of human TF, flow restriction leads to DVT with fibrin deposition in the inferior vena cava, though thrombus growth proceeds at a slower rate compared to normal TF expression [36]. On the other hand, coagulation proteins are expressed in the arteries affected by atherosclerosis, which enhances the inflammatory and immunologic processes typical of atherosclerosis [5, 37].

Microparticles, the membrane blebs released into blood by activated cells, that are associated with several vascular diseases, in particular CHD, could be another potential mechanism linking atherothrombosis and VTE as for example carriers of TF [38]. There have been reports showing an increased number of monocyte-derived TF-positive microparticles in both first and recurrent DVT compared to normal individuals [39]. Moreover, recent evidence indicates that microparticles, mainly platelet-derived, contribute to the development of damaged vessel wall-induced arterial thrombi by the induction of increased platelet and fibrin deposition on human atherosclerotic arteries [40].

Increased oxidative stress is known to represent a common denominator in many aspects of cardiovascular diseases including atherosclerosis, which appears to be implicated also in all types of thrombosis [41]. The augmented generation of reactive oxygen species from a number of sources including mitochondrial electron transport chain, NADPH oxidase, xanthine oxidase, and lipoxygenase/cyclooxygenase has been shown in varicose veins [42], suggesting that this mechanism might link all types of thrombosis as first suggested more than ten years ago [43].

A novel prothrombotic mechanism linking arterial and venous thrombosis represents prothrombotic fibrin clot phenotype characterised by the formation of more compact and highly branched fibrin fibre networks with lower clot permeability and prolonged lysis time [44, 45]. This kind of clots, with altered fibrin structure and function determined by genetic and environmental factors, has been reported in the plasma of patients with acute or prior $\mathrm{MI}$, ischaemic stroke, as well as those with prior idiopathic VTE $[44,45]$.

Nowadays, most prothrombotic mechanisms perceived as typically venous or arterial could be implicated in thrombosis in general, although their relative contribution varies at all stages of VTE and atherosclerosis. Much experimental effort is needed to clarify complex interactions between several potential cellular and plasmatic candidates as drivers of both types of thrombosis.

\section{PRACTICAL IMPLICATIONS}

The concept of similar pathogenesis of arterial and venous thromboembolism has multiple important implications in everyday practice. Firstly, patients with VTE should be thoroughly examined for the presence of not clinically overt atherosclerosis in order to identify subjects requiring additional management encompassing at least a modification of their risk profile. Secondly, some drugs used in cardiovascular disease could be effective in reducing the risk of VTE. The best example are statins, which produce a number of antithrombotic effects largely independent from cholesterol-lowering actions, including reduced TF expression, up-regulation of thrombomodulin expression on endothelial cells, attenuated platelet activation and increased fibrin susceptibility to lysis [46]. In 2009, it was demonstrated that rosuvastatin administered to healthy subjects with LDL cholesterol $<3.4 \mathrm{mmol} / \mathrm{L}$ and slightly elevated CRP > $2 \mathrm{mg} / \mathrm{L}$ can reduce the risk of symptomatic VTE, in particular DVT, by $43 \%$ within a two-year follow-up [47]. Meta-analysis of available studies, mainly observational, confirmed that various statins share the potential to reduce the risk of VTE [48].

Another example of a medication potentially effective in coronary artery disease and VTE appears to be aspirin, a cornerstone of secondary prevention in atherothrombosis. Recently, aspirin has been demonstrated to be effective in the secondary prevention of VTE [49]. Despite evidence suggesting the antithrombotic effects of aspirin beyond platelet cyclooxygenase inhibition, including acetylation of proteins like fibrinogen involved in haemostasis [50], in 2012 the first double-blind randomised placebo-controlled WARFASA study by Becattini et al. [49] provided a methodologically sound proof of this concept. It was shown that in patients with first-ever unprovoked VTE who had completed 6-18 months of oral anticoagulant treatment, aspirin given for a median period of 23.9 months reduces recurrent VTE compared to a placebo (HR 0.55; 95\% Cl 0.33-0.92) [49]. Based on this data, aspirin might be considered as a low cost and practical therapeutic option for the extension of secondary prophylaxis of unprovoked VTE in association with a well established MI or stroke prevention.

Similarly, anticoagulant agents, in particular warfarin, are able to reduce atherothrombotic events [51]. Warfarin, a primary agent used in the secondary prevention of VTE, has been shown to decrease the risk of recurrent $\mathrm{Ml}$ in stable angina patients, and it is used now particularly in patients with atrial fibrillation and CHD with or without low-dose aspirin [51].

Another practical implication of the association between various thromboses is the observation that lifestyle modifications are frequently equally effective in the prevention of VTE and atherothrombosis so physicians should encourage patients to adhere to them. For example, regular sports activities as 
well as increased fruit consumption can decrease the risk of arterial, as much as of venous, thrombosis [52, 53].

\section{CONCLUSIONS}

Despite several unresolved issues, growing clinical evidence suggests that thromboembolic events place the affected subject in a risk group prone to experience a new episode not only in the same kind of vessels. It is likely that the total thrombotic risk should be assessed to administer the optimal antithrombotic management in patients following either an arterial or a venous thromboembolic episode. In the era of personalised medicine, most experts recommend that patients following $\mathrm{MI}$, ischaemic stroke or any other arterial thrombosis episode should be assessed for the risk of VTE and vice versa. In everyday practice, this view requires for example to consider PE always in a patient post MI who complains of dyspnoea, chest dyscomfort or refractory cough, or to exclude DVT in a CHD patient with calf pain and/or oedema. With the prevalence of 1 to 1,000 middle-aged people and a $15 \%$ death rate in $\mathrm{PE}$, VTE is much more common also among patients suffering from cardiovascular disease than might have been expected [54].

Large prospective follow-up studies are needed to address the pivotal issue as to whether early initiation of anticoagulation in VTE patients confers a lower risk of not only MI, but also progression of atherosclerotic vascular disease. Further basic science research on the diverse associations between blood coagulation and atherosclerosis is needed. Its outcome might help optimise cardiovascular risk assessment in VTE subjects.

\section{Acknowledgements}

This work was supported by a grant of the Jagiellonian University (no K/ZDS/002936, to A.U.).

\section{Conflict of interest: none declared}

\section{References}

1. Lijfering WM, Flinterman LE, Vandenbroucke JP et al. Relationship between venous and arterial thrombosis: a review of the literature from a causal perspective. Semin Thromb Hemost, 2011; 37: 885-896.

2. Butenas S, Orfeo T, Mann K. Tissue factor in coagulation Which? Where? When? Arterioscler Thromb Vasc Biol, 2009; 29: 1989-1996.

3. Silvain J, Collet J-P, Nagaswami C et al. Composition of coronary thrombus in acute myocardial infarction. J Am Coll Cardiol, 2011; 57: 1359-1367.

4. Mazur P, Sobczyński R, Zạbczyk M et al. Architecture of fibrin network inside thrombotic material obtained from the right atrium and pulmonary arteries during embolectomy: flow and location matter. J Thromb Thrombolysis, 2013; 35: 127-129.

5. Kleinegris MC, Ten Cate-Hoek AJ, Ten Cate H. Coagulation and the vessel wall in thrombosis and atherosclerosis. Pol Arch Med Wewn, 2012; 122: 557-566.

6. Prandoni P, Bilora F, Marciori A et al. An association between atherosclerosis and venous thrombosis. N Engl J Med, 2003; 348: 1435-1441.

7. Becattini C, Agnelli G, Prandoni P et al. A prospective study on cardiovascular events after acute pulmonary embolism. Eur Heart J, 2005; 26: 77-83
8. Prandoni P, Ghirarduzzi A, Prins MH et al. Venous thromboembolism and the risk of subsequent symptomatic atherosclerosis. J Thromb Haemost, 2006; 4: 1891-1896.

9. Bova C, Marchiori A, Noto A et al. Incidence of arterial cardiovascular events in patients with idiopathic venous thromboembolism. A retrospective cohort study. Thromb Haemost, 2006; 96: 132-136.

10. Sorensen HT, Horvath-Puho E, Pedersen L et al. Venous thromboembolism and subsequent hospitalization due to acute arterial cardiovascular events: a 20 year cohort study. Lancet, 2007; 370 : 1773-1779.

11. Young L, Ockelford P, Milne D et al. Post-treatment residual thrombus increases the risk of recurrent deep vein thrombosis and mortality. J Thromb Haemost, 2006; 4: 1919-1924.

12. Becattini C, Vedovati MC, Ageno W et al. Incidence of arterial cardiovascular events after venous thromboembolism: a systematic review and meta-analysis. J Thromb Haemost, 2010; 8: 891-897.

13. Lowe GDO. Arterial disease and venous thrombosis: are they related, and if so, what should we do about it? J Thromb Haemost, 2006; 4: 1882-1885.

14. Lind C, Flinterman C, Enga $\mathrm{K}$ et al. Impact of incident venous thromboembolism on future risk of arterial thrombotic disease. ISTH Congress 2013, Abstract OC 07.1.

15. Agnelli G, Becattini C. Venous thromboembolism and atherosclerosis: common denominators or different diseases? J Thromb Haemost, 2006; 4: 1886-1890.

16. Eliasson A, Bergqvist D, Bjorck $\mathrm{M}$ et al. Incidence and risk of venous thromboembolism in patients with verified arterial thrombosis: a population study based on 23,796 consecutive autopsies. J Thromb Haemost, 2006; 4: 1897-1902.

17. Reich LM, Folsom AR, Key NS et al. Prospective study of subclinical atherosclerosis as a risk factor for venous thromboembolism. J Thromb Haemost, 2006; 4: 1909-1913.

18. van der Hagen PB, Folsom AR, Jenny NS et al. Subclinical atherosclerosis and the risk of future venous thrombosis in the Cardiovascular Health Study. J Thromb Haemost, 2006; 4: 1903-1908.

19. Piazza G, Goldhaber SZ, Lessard DM et al. Venous thromboembolism in patients with symptomatic atherosclerosis. Thromb Haemost, 2011; 106: 1095-1102.

20. Ageno W, Becattini C, Brighton T et al. Cardiovascular risk factors and venous thromboembolism: a meta-analysis. Circulation, 2008; 117: 93-102.

21. Deguchi H, Pecheniuk N, Elias D et al. High-density lipoprotein deficiency and dyslipoproteinemia associated with venous thrombosis in men. Circulation, 2005; 112: 893-899.

22. Di Minno MN, Tufano A, Guida A et al. Abnormally high prevalence of major components of the metabolic syndrome in subjects with early-onset idiopathic venous thromboembolism. Thromb Res, 2011; 127: 193-197.

23. Mahmoodi BK, Gansevoort RT, Veeger NJ et al; Prevention of Renal and Vascular End-stage Disease (PREVEND) Study Group. Microalbuminuria and risk of venous thromboembolism. JAMA, 2009; 301: 1790-1797.

24. Zacho J, Tybjaerg-Hansen A, Nordestgaard BG. C-reactive protein and risk of venous thromboembolism in the general population. Arterioscler Thromb Vasc Biol, 2010; 30: 1672-1678.

25. Heit JA, O'Fallon WM, Petterson TM et al. Relative impact of risk factors for deep vein thrombosis and pulmonary embolism: a population-based study. Arch Intern Med., 2002; 162: 1245-1248.

26. Zawilska K, Bała MM, Błędowski P et al. [Polish guidelines for the prevention and treatment of venous thromboembolism. 2012 update]. Pol Arch Med Wewn, 2012; 122 (suppl. 2): 3-74.

27. Ye Z, Liu EH, Higgins JP et al. Seven haemostatic gene polymorphisms in coronary disease: meta-analysis of 66,155 cases and 91,307 controls. Lancet, 2006; 367: 651-658.

28. Celinska-Lowenhoff M, Iwaniec T, Alhenc-Gelas M et al. Arterial and venous thrombosis and prothrombotic fibrin clot phenotype 
in a Polish family with type 1 antithrombin deficiency (antithrombin Krakow). Thromb Haemost, 2011; 106: 379-381.

29. Szymańska M, Alhenc-Gelas M, Undas A. Recurrent ischemic cerebrovascular events in a patient with type I antithrombin deficiency caused by $9788 \mathrm{G}>\mathrm{A}$ splice site mutation: a case report. Blood Coagul Fibrinolysis, 2013; 24: 213-215.

30. Mahmoodi BK, Brouwer JL, Veeger NJ, van der Meer J. Hereditary deficiency of protein $\mathrm{C}$ or protein $\mathrm{S}$ confers increased risk of arterial thromboembolic events at a young age: results from a large family cohort study. Circulation, 2008; 118: 1659-1667.

31. Arnson Y, Shoenfeld Y, Alon E, Amital H. The antiphospholipid syndrome as a neurological disease. Semin Arthritis Rheum, 2010; 40: 97-108.

32. Soltész P, Szekanecz Z, Kiss E, Shoenfeld Y. Cardiac manifestations in antiphospholipid syndrome. Autoimmun Rev, 2007; 6: 379-386.

33. Sare GM, Gray LJ, Bath PM. Association between hormone replacement therapy and subsequent arterial and venous vascular events: a meta-analysis. Eur Heart J, 2008; 29: 2031-2041.

34. Fuchs TA, Brill A, Wagner DD. Neutrophil Extracellular Trap (NET) impact on deep vein thrombosis. Arterioscler Thromb Vasc Biol, 2012; 32: 1777-1783.

35. Brill A, Fuchs TA, Savchenko AS et al. Neutrophil extracellular traps promote deep vein thrombosis in mice. J Thromb Haemost, 2012; 10: 136-144.

36. von Brühl ML, Stark K, Steinhart A et al. Monocytes, neutrophils, and platelets cooperate to initiate and propagate venous thrombosis in mice in vivo. J Exp Med, 2012; 209: 819-835.

37. Jawień J. Atherosclerosis in 2012: what is new? Pol Arch Med Wewn, 2012; 122: 170-173.

38. Budaj M, Poljak Z, Ďuriš I et al. Microparticles: a component of various diseases. Pol Arch Med Wewn, 2012; 122 (suppl. 1): 24-29.

39. Ye R, Ye C, Huang Y, Liu L, Wang S. Circulating tissue factor positive microparticles in patients with acute recurrent deep venous thrombosis. Thromb Res, 2012; 130: 253-258.

40. Suades R, Padró T, Vilahur G, Badimon L. Circulating and platelet-derived microparticles in human blood enhance thrombosis on atherosclerotic plaques. Thromb Haemost, 2012; 108: 1208-1219.
41. Szasz T, Thakali K, Fink GD, Watts SW. A comparison of arteries and veins in oxidative stress: producers, destroyers, function, and disease. Exp Biol Med (Maywood), 2007; 232: 27-37.

42. Guzik B, Chwała M, Matusik P et al. Mechanisms of increased vascular superoxide production in human varicose veins. Pol Arch Med Wewn, 2011; 121: 279-286.

43. Re G, Lanzarini C, Vaona I et al. Systemically circulating oxidative species in human deep venous thrombosis. Eur J Emerg Med, 1998; 5: 9-12.

44. Undas A, Ariëns RA. Fibrin clot structure and function: a role in the pathophysiology of arterial and venous thromboembolic diseases. Arterioscler Thromb Vasc Biol, 2011; 31: e88-e99.

45. Undas A. Acquired dysfibrinogenemia in atherosclerotic vascular disease. Pol Arch Med Wewn, 2011; 121: 310-319.

46. Undas A, Brummel-Ziedins KE, Mann KG. Statins and blood coagulation. Arterioscler Thromb Vasc Biol, 2005; 25: 287-294.

47. Glynn R, Danielson E, Fonseca F et al. A randomized trial of rosuvastatin in the prevention of venous thromboembolism. N Engl J Med, 2009; 360: 1851-1861.

48. Squizzato A, Galli M, Romualdi E et al. Statins, fibrates, and venous thromboembolism: a meta-analysis. Eur HeartJ, 2010; 31:1248-1256.

49. Becattini C, Agnelli G, Schenone A et al. Aspirin for preventing the recurrence of venous thromboembolism. NEngl J Med, 2012; 366: 1959-1967.

50. Undas A, Brummel-Ziedins KE, Mann KG. Antithrombotic properties of aspirin and resistance to aspirin: beyond strictly antiplatelet actions. Blood, 2006; 109: 2285-2292.

51. Hurlen M, Abdelnoor M, Smith P et al. Warfarin, aspirin, or both after myocardial infarction. N Engl J Med, 2002; 347: 969-974.

52. van Stralen KJ, Le Cessie S, Rosendaal FR, Doggen CJ. Regular sports activities decrease the risk of venous thrombosis. J Thromb Haemost, 2007; 5: 2186-2192.

53. Steffen LM, Folsom AR, Cushman M et al. Greater fish, fruit and vegetable intakes are related to lower incidence of venous thromboembolism: the longitudinal investigation of thromboembolism etiology. Circulation, 2007; 115: 188-195.

54. Di Minno MN, Tufano A, Ageno W et al. Identifying high-risk individuals for cardiovascular disease: similarities between venous and arterial thrombosis in perspective. A 2011 update. Intern Emerg Med, 2012; 7: 9-13. 